\title{
Bridging the gap of translation research in public health - from research to real world
}

\begin{abstract}
Objective: The reason behind heightened demand for translational research is a failure to achieve expected. Advances in the medical field have failed to bridge the gap between research results and real-world outcomes. The lack of effective research which can bridge the gap between community setting outcomes and results gained from small settings leads to the failure of interventions.
\end{abstract}

Study design: The current study is a systematic review.

Methods: Several peer reviewed articles published in the Web of Sciences Core Collection and Google Scholar database were chosen. The search words used were: "translational research", "real world", "public health", and "gap".

Results: This paper describes some effective approaches which have proven to be capable of bridging the gap between research and real-world effectiveness. These approaches include evaluation frameworks, evidence-based medicine, as well as prevention trial design.

Conclusion: Translational research plays a crucial role in achieving expected outcomes of any given intervention. These outcomes can only be achieved if a unified model or hybrid approach is used to address any given intervention of the program/ campaign. A hybrid model which can address the gaps at each level in this transition could prove to be the most promising strategy.

Keywords: translational research, bridging the gap, public health, research, real world
Volume 7 Issue 6 - 2018

\author{
Naiya Patel \\ Department of Public Health, Long Island University, USA
}

Correspondence: Naiya Patel, Department of Public Health, Long Island University, New York, 20 corbin ave, Floor I, Jersey City, NJ, 07306, USA, Tel 929-280-7280,

Email najya.patel2014@gmail.com

Received: November 30, 2018 | Published: December 07,

\section{Introduction}

The need for translational research has evolved over the past several years. ${ }^{1}$ There is, namely, a gap between research and practice which can be explained by several factors including lack of evidencebased recommendations, too few studies proving clinical validity and utility of the programs/products/interventions, and inadequate training for healthcare professionals. ${ }^{2}$ Regardless of advances in the medical field, with few exceptions we are unable to see expected outcomes in terms of intervention. Public health researchers and practitioners might want to come up with better approaches for interventions which are easy to translate into real-world settings. ${ }^{3}$ Due to such translational research gaps, expected results are not achieved. In January 2000, the U.S. Department of Health and Human Services launched Healthy People 2010, a comprehensive, nationwide, health promotion and disease prevention agenda. ${ }^{4}$ Only minimal progress has been made in the Healthy People 2010 goals regarding health disparities ${ }^{4}$ and it seems that flaws in translational research are one of the reasons behind its failure in achieving the expected objectives. It is evident that in order to address health disparities, the interventions suggested to solve them should be effective in real-world settings. However, inherent flaws seem to exist when researching topics targeted for public health interventions, making it inevitable that expected impacts are not achieved. ${ }^{5}$ Research by Kerner et al., ${ }^{6}$ states that "One of the greatest challenges facing health promotion and disease prevention is translating research findings into evidence-based public health and clinical practices that are actively disseminated and widely adopted". It is apparent that a gap exists between research and practice and that there is a need for better translational research strategies. Several suggestions have been made to bridge this gap between research and practice, one of which is the Participatory Research Approach. ${ }^{4}$ Participatory research includes participants and stakeholders in health intervention research, rather than considering them subjects of study. It helps to bridge the gap between research and practice. The practice-based evidence is another approach and discusses the participatory approach as one component along with efficacy and effectiveness, all of which are required to impact public health using tested interventions. 3 Another suggestion has been to move resources towards "the development and application of criteria and measures of external validity" as well as to "generate more practice-based evidence that explicitly addresses external validity and local realities". ${ }^{7}$ Existing multiple approaches to bridge this gap have proven to be effective. Some of these techniques, identified in the literature as successful in facilitating translational research, will be discussed in this paper. Significant approaches so far in the field are discussed below.

\section{Methods}

Several original research and review articles published in the Web of Sciences Core Collection as well as the Google Scholar database were reviewed. The search words used were: "translational research," "real world," "public health," and "gap." The articles were from peerreviewed journals. The current study is a systematic review study design.

\section{Results}

Approaches for bridging the translational gap- Overview of research studies 


\section{Approach I- evaluation framework}

This approach measures translation research through an evaluation framework. A paper by Brownson \& Simões ${ }^{8}$ describes the following evaluation approaches of a translational research process:

1. Process effectiveness, measured by taking into account the following factors:

a) Process: Process evaluation is performed to analyze the input and implementation of a particular program/policy or to keep track of it.

b) Impact: A subset of the outcome approach used to measure intermediate objectives of the program or intervention.

c) Outcome: Measures whether the expected result, such as mortality, morbidity or behavior change, has been achieved.

2. Cost-effectiveness analysis: Compares the total cost of an intervention with the quality of life achieved.

1. Potential hazard analysis: The benefits of intervention versus its harmful side effects. Helps to determine whether a particular intervention has more advantages over possible predicted unwelcomed outcomes by target population or not. This factor measures the overall beneficial aspects of an intervention.

2. Finally, a qualitative analysis used along with a quantitative analysis helps in achieving accurate research results.

All of the above-mentioned evaluation approaches will contribute to determining whether a research study is practical or not.

\section{Approach 2 - RE-AIM}

This approach, established by, ${ }^{9}$ provides a practical research tool to facilitate translational research through the framework of a webbased database named RE-AIM, which stands for:

a) Reach - Describes what percentage of the population participated, what percentage was excluded and how generalizable the sample was.

b) Efficacy/Effectiveness - Describes what impact the intervention had on all participants, the primary outcome, as well as positive or negative outcomes affecting the quality of life.

c) Adoption - Describes what percentage of intervention settings or stakeholders were included or excluded and how generalizable they were.

d) Implementation - Describes the setting level, i.e., to what extent each component of the intervention was delivered as expected by staff.

e) Maintenance - Comprises:

i. Setting-The extent to which intervention becomes institutionalized or part of routine practice

ii. Individual - As per.' Addresses "The long-term effects of a program on individual outcomes six or more months after the most recent intervention contact."

This approach helps researchers in the early stages of study planning to predict values and optimize the study.

\section{Approach 3- Intervention design}

A paper by Stone et al., ${ }^{10}$ provides suggestions about which intervention trial design best suits relevant community settings. Depending on the context, appropriate intervention designs can bring about desired outcomes and the gap between efficacies to effectiveness filled. These include: a) Youth and school settings - Interventions focused on learning and behavioral issues like smoking cessation or methods to tackle depression are achieved more efficiently in such setting context. Interventions focused on physiologic results like losing weight through exercise will not be as effective as the former one in this intervention setting. Hence the success of a health intervention depends on the environment in which it is implemented.

b) Healthcare practice setting - Multidisciplinary involvement facilitates achievement of the desired outcome in such a context like an intervention addressing HIV prevention through condom use where office setting is combined with education. Such intervention setting would involve a health educator and social worker in a clinic of the general physician or gynecologist raising awareness about condom use which can help prevent HIV infections at first place. The Health interventions focused on multidisciplinary collaborations nurses, social workers and physicians, for example, will assist in achieving expected outcomes of interventions.

c) Work setting - Providing counseling to high-risk employees produces the best outcomes for the total employee population. As discussed before, health intervention components should be designed and planned to keep in mind the context of practice setting. An irrelevant intervention component might not efficiently help us achieving expected outcomes.

\section{Policy implications}

Due to complex policies and practices it is an ongoing challenge to bridge the gap between evidence and practice. ${ }^{11}$ Evidence-based research is considered to be the gold standard in health promotion, hence motivation towards it is inevitable. ${ }^{12}$ Also, the ultimate goal is to disseminate research interventions in order to achieve predicted outcomes. ${ }^{13}$ We need to focus more on sustainability in terms of organizational factors for any intervention in practice for better results. ${ }^{14}$ Certain translational models fail to address the complexity of public health policies. ${ }^{15}$ The Translational Health Education Research framework for health education researchers is one suggestion to address the problem. ${ }^{16}$ Such structures help in fostering translational efforts as well as encouraging interdisciplinary work for better outcomes. The application of translational research frameworks will help to create better interventions and train researchers in such a way that they are able to come up with results which can be effectively applied in the real world. ${ }^{17} \mathrm{~A}$ unified model which includes all the critical factors necessary for effective interventions should also be considered and applied. ${ }^{18}$

\section{Conclusion}

Research results achieved in confined settings are mostly not applicable to real-world settings. Since the real-world sample size is more heterogenous than homogenous the tested interventions of any public health program ${ }^{19}$ may not be able to achieve expected results when applied to sample size. The generalization of any results must be measured or tested in such a way as to measure achievement of the expected outcome. The transition from basic science to community setting has not been smooth so far. Translational research plays a crucial role in achieving the expected outcomes of any intervention. These outcomes can only be achieved if a unified model or hybrid approach to address any intervention of the program /campaign is used. A hybrid model which can address the gaps at each level in this transition could prove to be the most promising strategy. A single approach is less effective over multi-approach models to bridge the 
gap between research and real-world interventions. Implementing a single approach, whether it be RE-AIM, practical medicine or prevention trials, will only end up addressing micro-level, isolated issues. In order to achieve a one hundred percent result, all the voids in the translational research journey need to be filled and addressed in the best possible way. One suggestion would be to develop customized combinations of two or more approaches relevant to the ongoing intervention and which can be applied to achieve expected outcomes. Not only would this prove to be effective, it would also save human and monetary resources as well as viewed from a business point of view.

\section{Acknowledgments}

None.

\section{Conflicts of interest}

The Authors have no conflicts of interest.

\section{References}

1. Armstrong R, Waters $\mathrm{E}$, Roberts $\mathrm{H}$, et al. The role and theoretical evolution of knowledge translation and exchange in public health. $J$ Public Health (Oxf). 2006;28(4):384-389.

2. Glasgow RE1, Lichtenstein E, Marcus AC. Why don't we see more translation of health promotion research to practice? Rethinking the efficacy-to-effectiveness transition. American journal of public health. 2003;93(8):1261-1267.

3. Ammerman A, Smith TW, Calancie L. Practice-based evidence in public health: improving reach, relevance, and results. Annu Rev Public Health. 2014;35:47-63.

4. Cargo M, Mercer SL. The value and challenges of participatory research strengthening its practice. Аппи Rev Public Health. 2008;29:325-350.

5. Estabrooks PA, Gyurcsik NC. Evaluating the impact of behavioral interventions that target physical activity: issues of generalizability and public health. Psychology of Sport and Exercise. 2003;4(1):41-55.

6. Kerner J, Rimer B, Emmons K. Introduction to the special section on dissemination: dissemination research and research dissemination: how can we close the gap? Health Psychol. 2005;24(5):443-446.

7. Green LW, Glasgow RE. Evaluating the relevance, generalization, and applicability of research: issues in external validation and translation methodology. Eval Health Prof. 2006;29(1):126-153.
8. Brownson RC1, Simoes EJ. Measuring the impact of prevention research on public health practice. American journal of preventive medicine. 1999;16(3):72-79.

9. Dzewaltowski DA, Glasgow RE, Klesges LM, et al. RE-AIM: evidencebased standards and a Web resource to improve translation of research into practice. Annals of Behavioral Medicine. 2004;28(2):75-80.

10. Stone EJ, Pearson TA, Fortmann SP, et al. Community-based prevention trials: Challenges and directions for public health practice, policy, and research. Annals of epidemiology. 1997;7(7):S113-S120

11. Rychetnik L, Bauman A, Laws R, et al. Translating research for evidencebased public health: key concepts and future directions. J Epidemiol Community Health. 2012;66(12):1187-1192.

12. Van Egmond S, Bekker M, Bal R, et al. Connecting evidence and policy: bringing researchers and policy makers together for effective evidencebased health policy in the Netherlands: a case study. Evidence \& Policy: A Journal of Research, Debate and Practice. 2011;7(1):25-39.

13. Glasgow RE, Vinson C, Chambers D, et al. National Institutes of Health approaches to dissemination and implementation science: current and future directions. American journal of public health. 2012;102(7):12741281.

14. Scheirer MA1, Dearing JW. An agenda for research on the sustainability of public health programs. American Journal of Public Health. 2011;101(11):2059-2067.

15. Ogilvie D1, Craig P, Griffin S, et al. A translational framework for public health research. BMC public health. 2009;9(1).

16. Mata HJ1, Davis S. Translational health research: perspectives from health education specialists. Clinical and translational medicine. 2012;1(1):27.

17. Shiramizu B, Shambaugh V, Petrovich H, et al. Leading by Success: Impact of a Clinical and Translational Research Infrastructure Program to Address Health Inequities. Journal of Racial and Ethnic Health Disparities. 2016:1-9.

18. Estape ES, Mays MH, Harrigan R, et al. Incorporating translational research with clinical research to increase effectiveness in healthcare for better health. Clinical and translational medicine. 2014;(1):20.

19. Healthy People - Healthy People Homepage. 2018. 